\title{
Methodical Bases for Developing Predictive Scenarios of Agribusiness
}

\author{
Andrey Nikolaevich Baidakov ${ }^{1}$, Natalia Borisovna Chernobay ${ }^{1}$, Anton Vladimirovich Nazarenko ${ }^{1}$, Dmitry \\ Vasilevich Zaporozhets ${ }^{1} \&$ Ekatherine Gennadievna Sergienko ${ }^{1}$ \\ ${ }^{1}$ FGBOU VPO Stavropol State Agrarian University, Russian Federation \\ Correspondence: Andrey Nikolaevich Baidakov, Zootechnical lane, 12, Stavropol, 355000, Russian Federation.
}

Received: November 9, 2014 Accepted: December 4, 2014 Online Published: March 20, 2015

doi:10.5539/ass.v11n8p9

URL: http://dx.doi.org/10.5539/ass.v11n8p9

\begin{abstract}
Complexity of the agricultural business tasks, high dynamism and non-linear nature of the contemporary socio-economic processes which differs functioning of any industry are placing new requirements for predictive studies. The purpose of this study is to develop a set of methodological provisions for the construction of predictive scenarios of the agricultural business by identifying current trends, the impact factors of the environment and the interpretation of results forecasting and analytical calculations. This article considers the influence of climatic factors on the economic impact of the frumentaceous and the grape branches of agriculture. The system of economic and mathematical prognostics models of the main industrial indicators was developed. The methodology for scenario forecasting of indicators of frumentaceous production and vine growing was proposed based on the use of the influence of solar activity on agrobiological processes.
\end{abstract}

Keywords: agricultural business, forecasting, scenario forecast methodology, the trend - cyclical patterns, price, cost, cost effectiveness, the cycles of solar activity, grain production, viticulture, economic and mathematical models

\section{Introduction}

Today a variety of mathematical models is use to predict the performance of the agricultural business. Effectiveness of prognostic study depends primarily on the choice of indicators, the availability of the necessary information base used the information and analytical tools and skills of the researcher. An important place in the development of forecasting activity in relation to the agricultural business belongs to the practical demand forecasts obtained. It should be kept in mind essentially nonlinearity of processes under investigation, which creates serious difficulties in obtaining reliable results at medium and especially in long-term forecasting (Panasyuk et al., 2014).

Negotiation of these difficulties is strongly influenced on the modeling level of the investigated processes of agrarian business allowing use the best modern informative and theoretical and methodological endeavours. Because of the complexity of the investigated phenomena, usually chronological heterogeneity of information sets, the duration of the study period, and therefore the changes in trends in its different parts is nigh on impossible to adequately describe the actual processes of agrarian business only through the use of a single, quite difficult mathematical model. And here it should be kept in mind as the modeling of individual indicators of the agricultural business as their totality. It means that, to obtain simulation results having sufficiently high accuracy, it is expedient to use some system of mathematical models, having sufficient flexibility and adaptability to changes in both the modeling object and its environment.

\section{Methods}

The successful functioning of the agricultural business depends on many factors, but the most important and meaningful component of information-analytical support of the management of its sustainable development is the prediction of grain production and viticulture. Productivity, the gross yield, crop area, sales price, the cost of its production, sales revenue, profit, profitability are included to the most important indicators of the state and development of these industries (Renaud-Gentié et al., 2014).

The proposed method of forecasting performances of grain farming and viticulture are based on the use of multivariate complex of mathematical economic regression, trending and trend-cycle models, extrapolation and 
adaptive prediction of the main indicators such as productivity, cost, selling price, the value of cultural area (Chernobay, 2012).

Choice and use of a set of mathematical modeling tools in the forecast process depend on informative database and taking into account the need to harmonize the influence factors on grain production and viticulture. The simulation of performances for the development of grain production and vine growing intended for further prognostic studies should therefore take into account the requirements of flexibility and adaptability of the built models from the position of prediction. In addition it supposes to assure possibility of regular update models when new information related to the subject of prognostic studies. Transformation of used models also is available on the obtained results and their verification, as well as verification and interpretation of the forecast scenarios of grain production and viticulture development (Sergiyenko \& Atanasova, 2013).

\subsection{The Method of Constructing the Forecast Scenarios of Development of Grain Growing Industry}

The need to improve the quality of forecasting research in modern conditions is related to the increasing importance of the predictive function in the information-analytical support of the management process of enterprises and industries. The practical significance of prediction results to inform management decisions is largely determined by methodological base, corresponding to features of the object. Therefore a comparative analysis of diverse methods used in the prediction of the basic parameters of the grain sector, in terms of their compliance with industry specifics, was carried out in this study. Particular attention was paid to the non-linearity of the projected processes responsible for the inadvisability of setting of rigid cause-and-effect relationship of the past with the future in long-term forecasting because of the inevitability of change in the trends in the bifurcation points. At the same time the use of extrapolation in the short-term forecasting, as well as in solving the problems of technical and economic nature was justified. (Carruthers \& Vanclay, 2012).

Forecasting of grain production should be based on the general methodological principles such as continuity, relevance, consistency, focus and priority, optimality, balance and proportion, and etc. We consider that it is necessary to supplement it with the principles of cyclicity and poly variety that reflect the specific of agricultural production, primarily in terms of environmental influence. On the one hand this is because the cyclical nature of the agricultural operation, and on the other hand this is due to a high level of uncertainty associated with the effect on it of the many factors that can be grouped into two groups that is natural and man-made groups (Panasyuk et al., 2014).

As a key factor in the first group we propose to consider the influence of solar activity. As it appears from the results of research in geobiology and gelioklimatologiya, variations of solar activity are dominant in the formation of complex natural and climatic impacts in the agricultural sector. In particular it has been proved that changes in relative humidity, temperature and soil microflora depend on solar activity.

In addition methodological and practical advantage of using the solar activity as a determinant natural factor in the prediction is the property of its recurrence, measurable with the help of the Wolf numbers (W) and the presence of a sufficient amount of historical data.

Application of cyclic waves in prediction allows you to find more accurate values of yield and other indicators of grain production, as well as quantitatively into account the ratio of natural and anthropogenic impacts on the development of grain production for a long period of time (Baydakov et al., 2013).

To construct predictive scenarios of grain production after formalizing patterns of change in selected indicators is intended to establish a common prediction model of values of the grain production indicators, unifying and coherent on the entire range of the parameters used. Yield forecasting, cost, selling price and the cultivation area is determined by the settlement and expert manner with the use of formalized dependencies and forecast indicators of gross collection, profits, cost effectiveness and some others are found by calculations on the basis of the values of other parameters (Nazarenko \& Zvyagintsev, 2012).

According to the results of the study dependencies of factors influence on grain production, the impact of anthropogenic factors is described by a linear trend, and natural is described by cyclical component, and trend-cycle model is constructed on the base of all of it. As a key factor in the group of natural factors we offer to consider the impact of solar activity in prediction of the grain production development. This means that in addition to the formalization of this relationship, there is the need to harmonize anthropogenic impacts on grain production with cyclic changes in the level of solar activity (Untaru et al., 2012).

In the study there was carried out modernization and development of the existing mathematical models and algorithms for predicting the development of the grain industry, aimed at ensuring the implementation of multivariate scenario forecasting based on solar cycles. 
In our opinion the forecast scenarios of the grain production development are models of the future state describing the likely direction of development taking into account the impact of the major factors of predictive environment which may have a significant impact on the future states of both individual companies and the industry as a whole (Nikulina et al., 2013).

\subsection{Algorithm Scenario Forecasting the Development of Grain Production}

During the research we carried out the modernization and development of the existing mathematical models and algorithms of predicting of the grain industry development aimed at ensuring the implementation of multivariate scenario forecasting based on solar cycles.

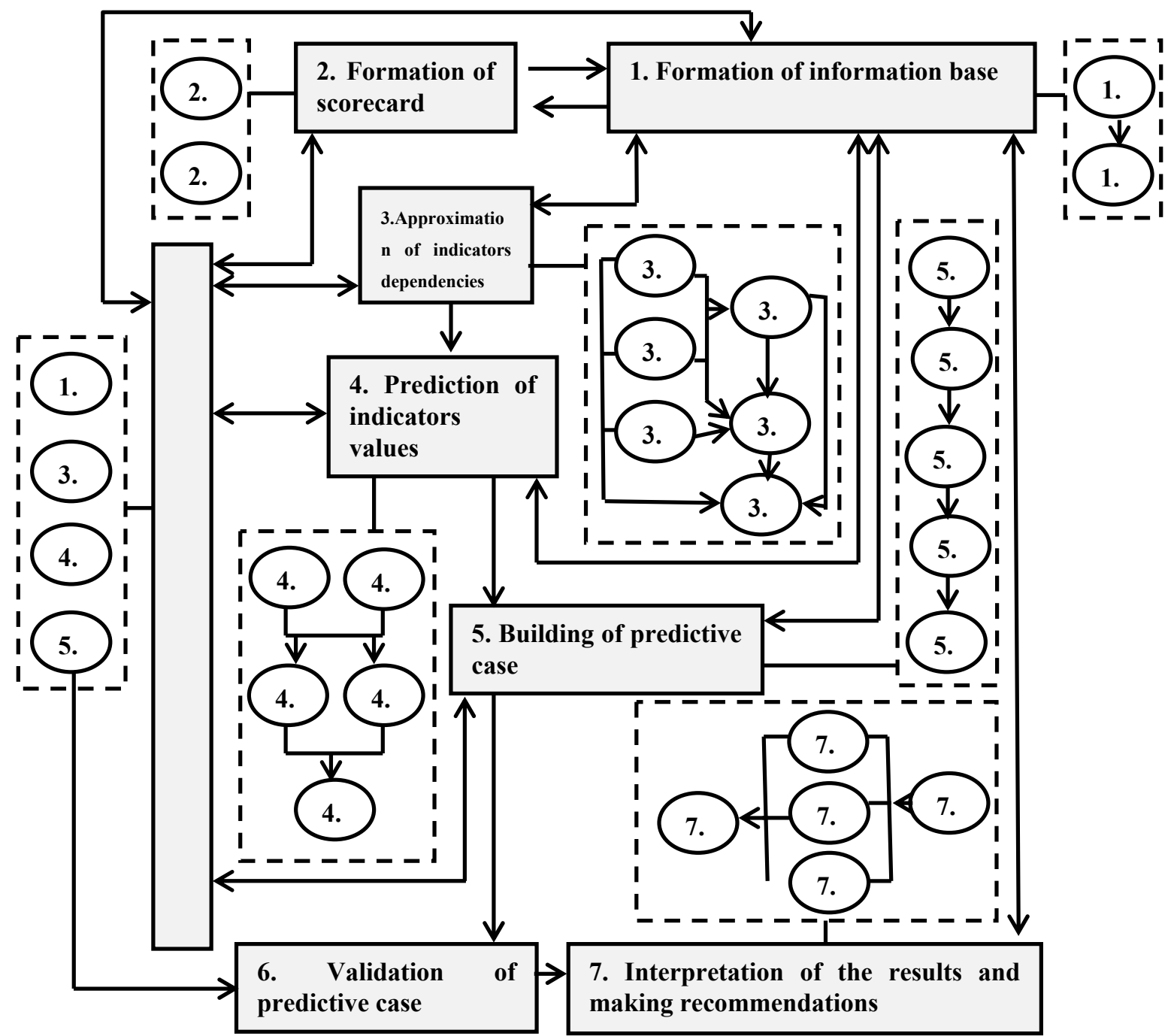

Figure 1. Algorithm of scenario forecasting of the grain production development

The algorithm of the proposed methodology of scenario performance forecasting of grain production which includes seven main stages and forming their blocks, has been shown in Figure 1, where:

(1.1) - factor variables;

(1.2) - the resulting variables;

(1.3) - verification of scorecard (logic, expert);

(2.1) - the primary information: statistics reports;

(2.2) - the post primary information: processing and calculation, the results of examinations, special literature;

(3.1) - construction of trends;

(3.2) - building regression equations; 
(3.3) - approximation of cyclic components;

(3.4) - the formation of trend-cycle models;

(3.5) - Implementation of "piecewise" approximation and "gluing";

(3.6) - determination calculated on the basis of the dependency patterns;

(3.7) - verification of the models (logical, expert);

(4.1) - determination of the period prediction;

(4.2) - the task of confidence probability;

(4.3) - prediction by model;

(4.4) - adaptive prediction;

(4.5) - building trust corridors;

(4.6) - Verification of forecasts (economic and logical evaluation of the results; retrospective forecasting; verification by analogy);

(5.1) - the definition of a set of the scenario indicators;

(5.2) -determination of values predicted va;

(5.3) - construction of scenario areas;

(5.4) - definition of scenario trajectories;

(5.5) - finding the quantitative characteristics for each scenario;

(5.6) - verification of forecast scenarios;

(7.1) - an expert interpretation, including

(7.2) - economic interpretation;

(7.3) - social explanation;

(7.4) - environmental interpretation;

(7.5) - development of recommendations.

In the forecasting process there was carried out simulation of grain production performance based on an integrated use of the following approximation methods: construction of trends (the crop area, yield, price, cost of production) and the regression equation (yield, price, cost); formalization of cyclical component (yield and price); formation of the trend-cycle model (yield, price); realization of "piecewise" approximation of dependencies and "bonding" (yield, price); calculation on the basis of the obtained dependences (gross fee revenue, profit margin) (Chernobay, 2012).

Cyclic and non-uniform effects of solar activity are taken into account through the use of various different phases of the cycle approximating functions and their subsequent agreement ("gluing").

Thus, modeling indicators of grain production was done to predict the development of grain production based on an integrated use of the following approximation methods: construction of trends (yield, price, cost of production) and the regression equation (price); formalization of cyclical component (yield and price); formation of the trend-cycle model (yield, price); realization of "piecewise" approximation of dependencies and "bonding" (yield, price). The proposed methodology of scenario forecasting of grain production performance has a number of distinctive features associated with a significant non-linearity of predicted processes. First of all, for each projected figure provided by the formation of adapted Instrumentation complex to the peculiarities of its changes (Baydakov et al., 2011).

\subsection{The Method of Constructing the Forecast Scenarios of the Development of Viticulture}

As for the viniculture, it is difficult object for prediction formed under the influence of a number of factors to ensure its normal functioning and development. (Frolov et al., 2013). To identify and examine the factors that have a direct impact on the grapes, it must be regarded as a system - ampelocenoz, artificially created community of grape plants, placed in optimal environmental conditions for his growing to produce grape and wine making products (Figure 2).

The principal difference between this scheme and the existing scheme is to allocate the impact of solar activity as a key natural factor. Also, this scheme establishes as a direct influence of natural factors on anthropogenic factors 
as a feedback that reflects the human impact (positive and negative) on nature, changing the basic state of ampelocenozes. In the course of the work we share the natural factors into two categories - abiotic and biotic groups, at this time territorial, climate factors and the impact of solar activity may be isolated in the abiotic group.

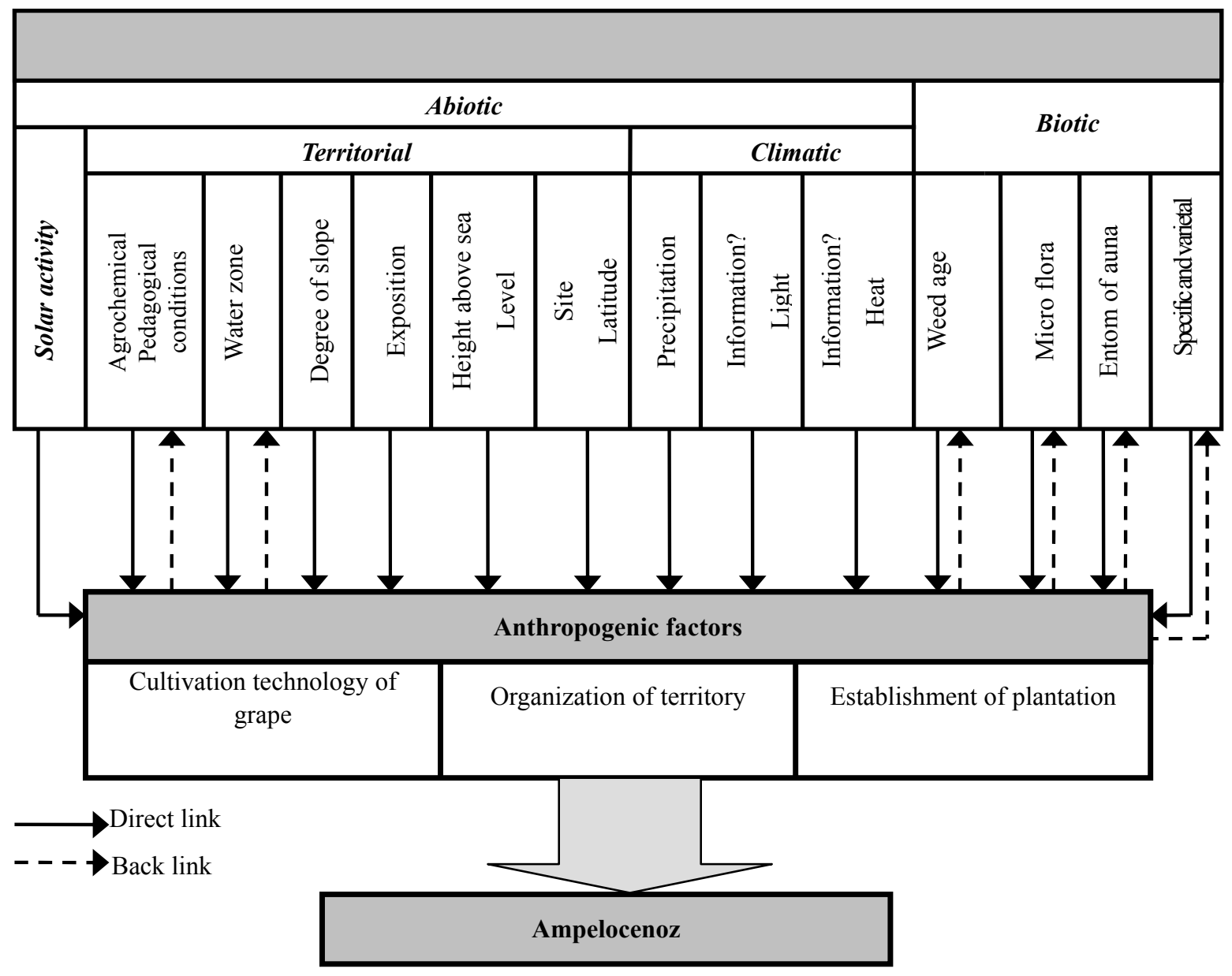

Figure 2. Factors identifying the productivity of ampelocenoz

The determining factors are the natural and climatic conditions, as a set of soil and climate, land reclamation, and other agro-meteorological characteristics of the area (Lee et al., 2011).

\subsection{Algorithm Study of Planned Development Scenarios Viticultural Industry}

Mathematical modeling methods are the most common methodological tools of prediction. Using mathematical methods allows us to describe complex economic processes in mathematical language, to calculate the value and results of the interaction of the studied factors and the object of forecasting, as well as to determine the extent of these processes.

The main feature of multivariate dynamic models is that within it the formation of the system is analyzed not only under the influence of the time factor, but also the relationship with other factors having a significant impact on the object in question. This fact contributes to a deeper analysis of the predictive object on the subject of establishing a functional relationship with the system of selected factors. The construction of this type of models is based on the use of regression analysis. These models have been widely applied in the prediction of the main indicators characterizing the development of wine-growing industry, and to establish a quantitative impact on it by factors of directly predictive environment (Bannikova et al., 2011). 


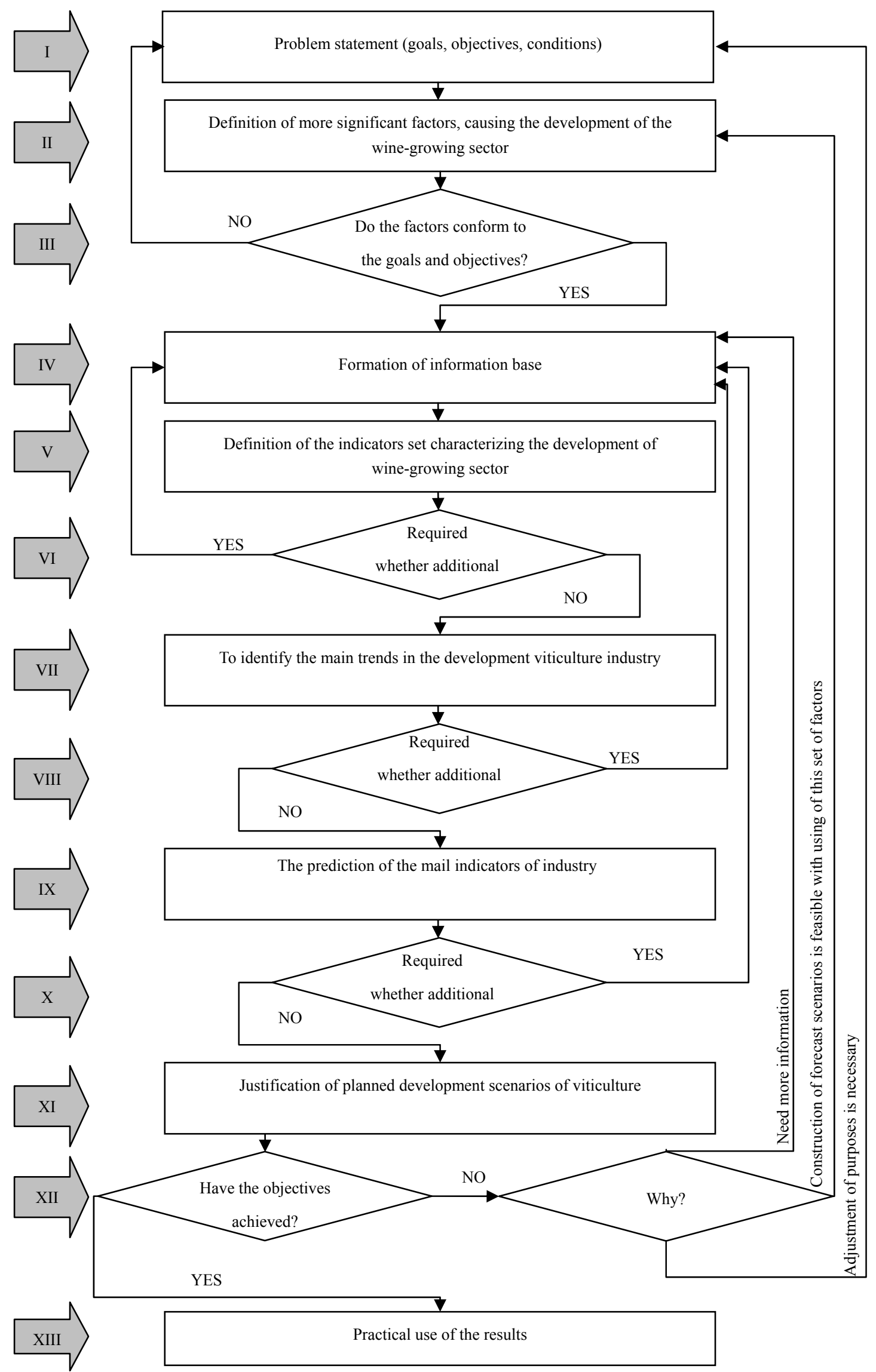

Figure 3. Algorithm of planned development scenarios of viticultural industry 
Forecast scenarios of viticulture include predictive models describing the likely directions of development, taking into account the impact of the major factors of predictive environment and a set of managerial actions aimed at minimizing the consequences of crisis situations and improve the efficiency of both individual enterprises and the industry as a whole vineyard.

In relation to the object under study forecasting is necessary to carry out with the help of a variety of ways. The above methods should be used in combination synthesizing the results of a comprehensive solution to the problem, forming a complete comprehensive forecast of the grape and wine making industry (Baydakov \& Nazarenko, 2011).

On the basis of research in the field of forecasting the development of wine-growing industry, we proposed an algorithm for study of planned viticulture development scenarios (Figure 3), which includes thirteen stages.

The initial phase of forecast scenarios construction is the Problem statement (goals, objectives, conditions). Further, according to the goals and objectives within acceptable conditions it is necessary to define a set of factors that determines the development of the viticulture industry.

In the third step it is checked for compliance with the resulting set of factors to set goals in the case of a negative result initial conditions is corrected, and in the case of a positive result - the algorithm proceeds to the next stage - the creation of the information database (stage IV). In the fifth stage it is necessary to select the main indicators characterizing the development of wine-growing industry on the basis of a specific set of factors and the information on them. In the case of the establishment of information about (step VI), that a given amount of information is not sufficient for the implementation of these measures, it is advisable to go back to recruiting of information base, otherwise you must go to identify the main trends of the industry development on the received parameters (step VII). In the case of need the information base is corrected, and then in the next step, a forecast of selected indicators reflecting the development of wine-growing industry is determined (Altukhova et al., 2013).

On the tenth stage when unsatisfactory results of prediction are obtained decision on additional research on information maintenance process is made. If the results reflect the identified trends of the development, within the limits of the algorithm we proceed to the justification of planned development scenarios of viticulture industry. Thus, for achieving the goals and objectives of the last step is to transfer the results to solve specific applied problems. However, if the goals are not achieved then depending on the reason you need to return to the earlier stages of the algorithm for further work on the problem set (Gerasimov et al., 2013).

\section{Results}

The resulting performance projections of grain production and viticulture allow on their basis to build scenarios for development: realistic, pessimistic and optimistic.

Forecasting of grain production is not limited to obtaining point forecasts. On their basis their confidence intervals is built for a given confidence level for the various years of the pre-emption period, which form a trust corridors. Based on the use of these corridors forecast region and sub region for each forecast scenario are constructed. Within each of the forecast subdomains the scenario trajectories are selected representing an aggregate value of some projected figures for all years of the period of pre-emption, which belong to the group of forecast scenarios subdomain with certain specified characteristics on the basis of these or other reasons (Nikitenko, 2012 ). The partition of trust corridor was completed by calculated expert method to determine the limits of scenario areas in this study on the following principle. Region is fit in with realistic scenario forecasts, the center line of which is the point forecast for a given period of pre-emption, and its width is equal to the minimum width of the confidence corridors of retrospective prediction.

Predictive subarea, located between the upper limit of a realistic scenario forecasting to the upper limit of the "trust corridor", is an area, which determines the number of possible optimistic scenario forecasts; and from the lower edge of a realistic prediction to the lower limit of the confidence interval this subarea is area of pessimistic forecasts of the grain production development.

Prediction of acreage values, selling price of winter wheat and its cost, formed under the influence of natural and anthropogenic factors, made a complex of other important problems solved in the course of the study, during the construction of the forecast scenarios for the grain production development.

Selection of specific forecast trajectories for all considered parameters within the respective forecast subarea was performed with use of computational and expert methods.

Thus constructed aggregate of forecast scenario trajectories for all indicators of research formed the forecast 
scenarios for grain production (Trukhachev et al., 2011).

Thus, the primary management task, the solution of which will contribute to the development of the industry and growth of its financial and economic situation, - is the implementation of constructive activities to achieve (or even exceed) the parameters defined by the area of optimistic scenario trajectories, and also leveling activity for elimination of negative preconditions for the implementation of the pessimistic scenario trajectories of the grain production development based on use of the prediction results.

As the scenario forecasts of the viticulture development we mean the area are formed by the boundaries that identify generated script. To determine the "scenario Borders" the width separation of the "trust corridor" was made into four equal parts, thus, in our opinion and on the base of the historical information study, the range of realistic scenarios should be wider than the other, so the two central parts of the segment are realistic forecast scenario. All variation of data flowing into the sector, refers to a realistic scenario forecasts. Predicted area located from the realistic to the upper limit of the "corridor" is an optimistic scenario, and the lower edge of the sector from a realistic prediction to the lower limit of the confidence interval is a pessimistic scenario.

\section{Discussion}

In our opinion the forecast scenarios for the development of viticulture and grain production are models of the future state of the agricultural business, describing the likely direction of development, taking into account the impact of the major factors of predictive environment, which may have a significant impact on the future status of both individual businesses and business as a whole (Bannikova, 2004).

With the help of scenarios it is possible to more fully and accurately determine the prospects of agricultural business development in the current economic situation, as with the dissimilarity of the impact of various factors affecting the rate of the industry development. Moreover it is possible to predict in advance the dangers arising from the impact of inefficient management, unfavorable development of the macroeconomic situation, and also in terms of force majeure occurring natural disasters.

Generated methods of prognostic study of agrarian business processes can quantify into account the impact of both natural and anthropogenic factors on their performance, not only in the medium, but also in the long-term (Shi et al., 2013).

Therefore, scientific and practical need to build predictive scenarios of the agricultural business based on the use of advanced information and analytical technology is the modern nature that determined the relevance, scientific logic and content of this research.

\section{Conclusion}

Studies in the field of teaching of the forecasting trends basics in the industry of Agribusiness allow us to make the following conclusions:

1) The terms of forecasting in agricultural business are substantially different from this kind of activity in other sectors of the economy and are characterized by specific features, based on the laws of its development, hence the need of developing specific approaches for using of prognostic tools. This is due to the specific features of the industry, and firstly, the influence of environmental conditions, which can be expressed in an integrated manner via its impact on the formation of the solar activity yield which determines the biological and climatic conditions for cultivation of agricultural crops.

2) The algorithm of study for the grain industry and wine forecast scenarios is quite versatile and can be used after appropriate adaptation for the predictive studies at various levels of the agricultural business.

3) Investigations showed that there are law-governed periods of prevailing alternating influence of anthropogenic and natural factors on economic performance in the agricultural business, hence the need of elaboration for different approaches to the management of the development of the industry and individual enterprises.

4) The state of Agribusiness is currently characterized by a lack of positive trends - the efficiency of agricultural enterprises decreases; competition from foreign producers increases; a significant portion of the national technical and technological support of the industry has been eliminated. Analysis of the situation leads to the conclusion about the high risks inherent in this type of activity, low efficiency of government support because of a lack of effective mechanisms for prediction of the future state of the main trends in the industry.

5) There are three main directions of development of the situation in the agricultural business, which leads to the justification of the development forecast scenarios of the industry: pessimistic, realistic and optimistic. This implies the need to develop appropriate strategies to reduce the impact of possible negative consequences and 
enhance the impact of the use of positive trends.

6) In order to reduce the risk of occurrence of events in the adverse scenario of the agricultural business and maximize use of the opportunities that are favorable for the production periods of the solar activity cycle to business entities is recommended:

- To use the proposed methodology in prediction of the values of the agricultural business indicators, it will allow to consider the main trend of its development and the cyclical nature of the changes under the influence of natural factors in complex and, thereby, to increase the assurance of the predictions.

- To use the proposed approaches and methods for forecasting the development of the agricultural business at various levels of the industry management (company, district, region, state), and also other sectors of the economy; it will allow to make informed pre-empt management decisions, to carry out the actions based on these decisions and adapted under realizable plan, to optimize the resource expenses and production processes via bringing into accordance with the phases of the solar activity cycle.

7) Reasonable scenarios of agricultural business should be used at different levels:

- Preparation of state development programs;

- Coordination of the implementation of measures to support the agricultural business via the distribution of funding by the articles;

- Development of management actions adapted to the implemented plan and aimed at the sales of products, adaptation of production capacity, changes in technology, expenses optimization and processes, increasing the range and quality of products in order to expand into new markets.

\section{Acknowledgments}

We express our gratitude to the doctor of economic sciences, Doctor of Agricultural Sciences, Professor, Corresponding Member of the RAAS; Honored Scientist of the Russian Federation, the rector of the Stavropol State Agrarian University Trukhachev Vladimir Ivanovich for their assistance and support to the research activities and professional development opportunities. Your theoretical knowledge and practical experience, ability and skills of to be a mentor make possible successfully to implement our plans in life.

\section{References}

Altukhova, L. A. et al. (2013). The description and analyses of grain production dynamic in Stavropol territory The current problems of agribusiness management. Monograph.

Bannikova et al. (2011). The methodological approaches for construction of agricultural development programs. Economics of agricultural and processing enterprises, 8, 20-23.

Bannikova. (2004). The specifics of strategic planning of agrarian production. Advances in science and technology of agriculture, $11,15$.

Baydakov et al. (2011). Scenario forecasting as a necessary component of the risk management system. Herald of Stavropol. AIC, 3, 55-58.

Baydakov et al. (2013). The construction of the forecast scenarios of the grain production development. Multidisciplinary Network electronic scientific journal of the Kuban State Agrarian University, 94, 784-794.

Baydakov, \& Nazarenko. (2011). Scenario forecasting in managing agricultural economies. Bulletin of the Institute of Friendship of Peoples of the Caucasus "Theory of Economics and Management of the national economy", 4(20), 21-26.

Carruthers \& Vanclay. (2012). The intrinsic features of Environmental Management Systems that facilitate adoption and encourage innovation in primary industries. Journal of Environmental Management, 110, 125-134. http://dx.doi.org/10.1016/j.jenvman.2012.06.003

Chernobay. (2012). Scientific approach to environmental management. Simulation of industrial processes and information systems, 244-246.

Chernobay. (2012). Using the scientific principles in management of agrarian sector. Bulletin NCSTU, 13, 205-208.

Frolov et al. (2013). Long-term forecast of the production capacity of high-tech industries in the Russian Federation. Studies on Russian Economic Development, 24(3), 229-236. http://dx.doi.org/10.1134/S10757 00713030040 
Gerasimov et al. (2013). Modeling and forecasting of key indicators of socio-economic development of traditionally agrarian regions. World Applied Sciences Journal, 27(10), 1282-1287.

Lee et al. (2011). Bio hydrogen development in United States and in China: An input-output model study. International Journal of Hydrogen Energy, 36(21), 14238-14244. http://dx.doi.org/10.1016/j.ijhydene.2011. 05.084

Nazarenko \& Zvyagintsev. (2012). Scenario forecast of the development of socio-economic systems. Multidisciplinary Network electronic scientific journal of the Kuban State Agrarian University, 84, 575-587.

Nikitenko. (2012). The validation of predictive scenarios of the grain production development. Master's thesis for the degree of economic sciences candidate. Stavropol State Agrarian University. Stavropol.

Nikulina et al. (2013). The formation of priority directions of social and economic development of the region. World Applied Sciences Journal, 22(5), 608-615.

Panasyuk et al. (2014). Modified index method in scenarios of regional socio-economic development. Mediterranean Journal of Social Sciences, 5(18), 331-334.

Panasyuk et al. (2014). Regional multicultural community: Problems of life quality estimation. Mediterranean Journal of Social Sciences, 5(18), 323-326.

Renaud-Gentié et al. (2014). Choosing the most representative technical management routes within diverse management practices: Application to vineyards in the Loire Valley for environmental and quality assessment. European Journal of Agronomy, 56, 19-36. http://dx.doi.org/10.1016/j.eja.2014.03.002

Sergiyenko \& Atanasova. (2013). The cyclical fluctuations as an integral laws of the economic situation development. Information systems and technology as a factor of regional economy development, 144-146.

Shi et al. (2013). Impacts of cultivated land reclamation on the climate and grain production in Northeast China in the future 30 years. Advances in Meteorology. http://dx.doi.org/10.1155/2013/853098

Trukhachev et al. (2011). The strategic planning in agriculture. Theory and practice. Stavropol.

Untaru et al. (2012). Artificial neural networks for sustainable agribusiness: A case study of five energetic crops. Agrociencia, 46(5), 507-518.

\section{Copyrights}

Copyright for this article is retained by the author(s), with first publication rights granted to the journal.

This is an open-access article distributed under the terms and conditions of the Creative Commons Attribution license (http://creativecommons.org/licenses/by/3.0/). 